\title{
Distance-2 MDS codes and latin colorings in the Doob graphs
}

\author{
Denis S. Krotov • Evgeny A. Bespalov
}

Received: 2016-11-15 / Accepted: 2018-07-11

\begin{abstract}
The maximum independent sets in the Doob graphs $D(m, n)$ are analogs of the distance-2 MDS codes in Hamming graphs and of the latin hypercubes. We prove the characterization of these sets stating that every such set is semilinear or reducible. As related objects, we study vertex sets with maximum cut (edge boundary) in $D(m, n)$ and prove some facts on their structure. We show that the considered two classes (the maximum independent sets and the maximum-cut sets) can be defined as classes of completely regular sets with specified 2-by-2 quotient matrices. It is notable that for a set from the considered classes, the eigenvalues of the quotient matrix are the maximum and the minimum eigenvalues of the graph. For $D(m, 0)$, we show the existence of a third, intermediate, class of completely regular sets with the same property.
\end{abstract}

Keywords Doob graph · Maximum independent set · Maximum cut · MDS code $\cdot$ Latin hypercube $\cdot$ Equitable partition $\cdot$ Completely regular set

Mathematics Subject Classification (2010) 05B15 $\cdot 94 \mathrm{~B} 25$

This is the accepted version of the paper in Graphs and Combinatorics, Vol. 34, Iss. 5, pp. 1001-1017, 2008; doi https://doi.org/10.1007/s00373-018-1926-4 C) Springer Japan KK, part of Springer Nature 2018

The work was funded by the Russian Science Foundation (grant No 14-11-00555).

D. Krotov

Sobolev Institute of Mathematics, pr. Akademika Koptyuga 4, Novosibirsk 630090, Russia Tel.: +7-383-329-75-42

E-mail: krotov@math.nsc.ru

orcid 0000-0002-8516-755X

E. Bespalov

Sobolev Institute of Mathematics, pr. Akademika Koptyuga 4, Novosibirsk 630090, Russia E-mail: bespalovpes@mail.ru orcid 0000-0001-9484-0091 


\section{Introduction}

In this paper, we characterize the maximum independent sets in the Doob graphs. The Doob graph $D(m, n)$, as a distance-regular graph, has the same parameters as the Hamming graph $H(2 m+n, 4)$. As we will see, the maximum independent sets in the Hamming graphs share many properties with those sets in the Doob graphs. This conclusion was already suggested by the existence of the injective map from the class of maximum independent sets in $D(m, n)$ to the class of maximum independent sets in $H(2 m+n, 4)$ 11. However, there are some difficulties with the use of that injection for the characterization of the former class, and the authors of the current paper decided to use another way to prove the characterizing theorem, presented in Section 4.

The maximum independent sets in the Hamming graphs are studied in different areas of mathematics. In coding theory, they are the distance- 2 MDS codes (these codes do not correct any errors, but they are used in the construction of codes with larger code distance). In combinatorics, these sets are known as the latin hypercubes, multidimensional generalizations of the latin squares, which correspond to the case $n=3$ (one of the coordinate is usually considered as dependent from the others). In nonassociative algebra, an $n$-ary quasigroup is exactly a pair of a set and an $n$-ary operation over this set whose value table is a latin hypercube. Every maximum independent set in a Hamming graph is a completely regular code of radius 1 ; the nontrivial eigenvalue of this code is the minimum eigenvalue of the graph.

For fixed $q$, the class of the distance-2 MDS codes in $H(N, q)$ is described for $q \leq 4$. For $q \leq 3$, the description is rather simple as for each $n$ there is only one such set, up to equivalence. In the case $q=4$, there are $2^{2^{N+o(N)}}$ nonequivalent MDS codes in $H(N, 4)$; however, there is a constructive description of the class of these codes [12. Notably, the case $q=4$ is a special case for the Hamming graphs $H(N, q)$ from the point of view of algebraic combinatorics: $H(N, 4)$, $N \geq 2$ are the only Hamming graphs that are not defined as distance-regular graphs with given parameters. The distance-regular graphs with the same parameters as $H(N, 4)$ are the Doob graphs $D(m, n), 2 m+n=N$.

The main goal of the current research is to extend the characterization theorem 12 for the distance-2 MDS codes in $H(n, 4)=D(0, n)$ to the maximum independent sets in $D(m, n)$ with $m>0$. The characterization theorem is formulated in Section 4 and proven in Section 6. In conjunction with the results of [1], this result can also be considered as completing the characterization of the MDS codes in the Doob graphs (there is also an infinite class of MDS codes with distance coinciding to the graph diameter and a few distance- 3 and distance-4 codes [1]).

As an intermediate result, in Section [5] we prove a connection between properties of related objects, $2 \times \mathrm{MDS}$ codes, which can be defined as the sets with largest edge boundary (see Subsection [7.1). This result also generalizes its partial case $m=0$, considered in 9 .

In Section 7, we consider alternative definitions of MDS and $2 \times$ MDS codes in Doob graphs. In particular, we show that these two classes can be character- 
ized in terms of equitable 2-partitions with quotient matrices whose nontrivial eigenvalue is the minimum eigenvalue of the graph. In $D(m, 0)$, we construct an equitable partition that has intermediate parameters between MDS and $2 \times$ MDS codes (strictly speaking, the quotient matrix of a new equitable partition is the arithmetic average of two quotient matrices corresponding to an MDS code and to a $2 \times$ MDS code). The existence of such "intermediate" objects between MDS and $2 \times$ MDS codes is a new effect, which has no analogs in Hamming graphs.

The next two sections contain preliminaries and auxiliary facts.

\section{Preliminaries}

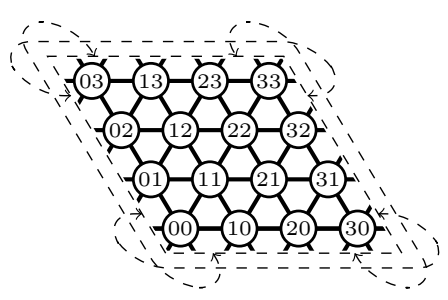

Fig. 1 The Shrikhande graph drown on a torus; the vertices are identified with the elements of $Z_{4}^{2}$

The Shrikhande graph Sh is the Cayley graph of the group $Z_{4}^{2}$ with the connecting set $\{01,10,11,03,30,33\}$ (the vertices of the graph are the elements of the group $Z_{4}^{2}$, which will be denoted $00,01,02,03,10, \ldots, 33$; two vertices are adjacent if and only if their difference belongs to the connecting set) see Fig. 1. The complete graph $K=K_{4}$ of order 4 is the Cayley graph of the group $Z_{2}^{2}$ with the connecting set $\{01,10,11\}$. The Cartesian product of $m$ copies of Sh and $n$ copies of $K_{4}$ will be denoted by $D(m, n)$. This graph is called a $D o o b$ graph if $m>0$, while $D(0, n)$ is a 4-ary Hamming graph. Note that $D(m, n)$ is a Cayley graph of $\left(Z_{4}^{2}\right)^{m} \times\left(Z_{2}^{2}\right)^{n}$, with the corresponding connecting set.

Given a graph $G$, by v $G$ we denote its set of vertices. Two subsets of v $G$ are said to be equivalent if there is a graph automorphism that maps one subset to the other.

An independent set of vertices of maximal cardinality, i.e. $4^{2 m+n-1}$, in $D(m, n)$ is called a distance- 2 MDS code (the independence number $4^{2 m+n-1}=$ ${ }_{\mathrm{v}} D(m, n) \mid / 4$ follows easily from the independence numbers 4 and 1 of Sh and $K$, respectively). The two inequivalent MDS codes in $D(1,0)$ are shown in Fig. 2

Lemma 1 If a subgraph $G$ of $D(m, n)$ is isomorphic to a Doob graph (with smaller parameters), then the intersection of an $M D S$ code with ${ }_{\mathrm{v}} G$ is an $M D S$ code in $G$. 
(a)

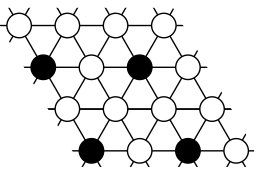

(b)

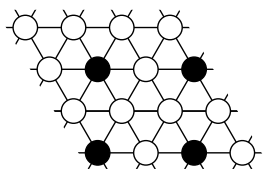

Fig. 2 All MDS codes in Sh, up to isomorphism

(a)

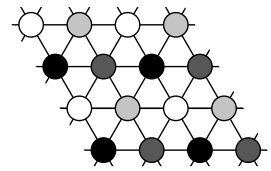

(b)

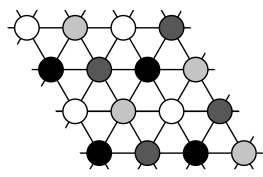

(c)

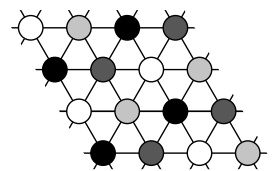

Fig. 3 All latin colorings of the Shrikhande graph, up to isomorphism

A function $f: \mathrm{v} D(m, n) \rightarrow \mathrm{v} K_{4}$ is called a latin coloring if the preimage of every value is an MDS code; i.e., no two neighbor vertices have the same colors. In the case $m=0$, the latin colorings are known as the latin hypercubes (if $n=2$, the latin squares) of order 4 . The case $m=1, n=0$ is illustrated in Fig. 3 ,

The graph $^{1}\left\{\left(y_{1}, \ldots, y_{m}, x_{1}, \ldots, x_{n}, x_{0}\right) \mid x_{0}=f\left(y_{1}, \ldots, y_{m}, x_{1}, \ldots, x_{n}\right)\right\}$ of a latin coloring $f$ is always an MDS code. Inversely, any MDS code in $D(m, n+1)$ is a graph ${ }^{1}$ of a latin coloring of $D(m, n)$ (however, the MDS codes in $D(m, 0)$ cannot be represented in such a manner). Moreover, if $f$ and $f^{\prime}$ are latin colorings of $D(m, n)$ and $D\left(m^{\prime}, n^{\prime}\right)$, then the set

$$
\left\{\left(\bar{y}, \bar{y}^{\prime}, \bar{x}, \bar{x}^{\prime}\right) \in \mathrm{v} D\left(m^{\prime}+m, n^{\prime}+n\right) \mid f(\bar{y}, \bar{x})=f^{\prime}\left(\bar{y}^{\prime}, \bar{x}^{\prime}\right)\right\}
$$

is an MDS code in $D\left(m^{\prime}+m, n^{\prime}+n\right)$. If $2 m+n>1$ and $2 m^{\prime}+n^{\prime}>1$, then the MDS code (1) is called reducible, as well as all codes obtained from it by coordinate permutation.

A set $M$ of vertices of $D(m, n)$ is called a $2 \times M D S$ code (two-fold MDS code) if every Shrikhande subgraph of $D(m, n)$ intersects with $M$ in 8 vertices that form two disjoint MDS codes in Sh (see Fig 4) and every clique of order 4 contains exactly 2 elements of $M$. The union of two disjoint MDS codes is always a $2 \times$ MDS code; such a $2 \times$ MDS code will be called bipartite. The complement $\mathrm{v} D(m, n) \backslash M$ of any $2 \times \mathrm{MDS}$ code $M$ is also a $2 \times \mathrm{MDS}$ code, which will be denoted by $\bar{M}$. However, it is not known if the complement of any bipartite $2 \times$ MDS code is also a bipartite $2 \times$ MDS code (the known solution 13 for $D(0, n)$ is far from being easy).

A connected component of a subgraph of $D(m, n)$ induced by a $2 \times$ MDS code $M$ will be called a component (of $M$ ). A $2 \times$ MDS code is called connected if it is a component itself. The union of (one or more) components of the same $2 \times$ MDS code will be referred to as a multicomponent.

A $2 \times$ MDS code is called decomposable (indecomposable) if its characteristic function can (cannot) be represented as a modulo-2 sum of two 0,1-functions

\footnotetext{
1 We will use this footnote mark to separate this notion from another meaning of the word "graph" (a pair of a set of vertices and a set of edges).
} 
(a)

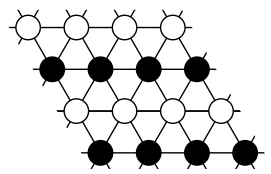

(b)

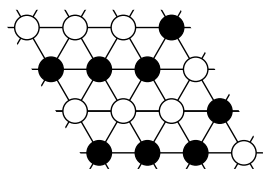

(c)

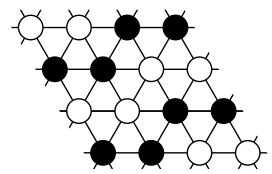

Fig. 4 All $2 \times$ MDS codes in the Shrikhande graph, up to isomorphism

in disjoint nonempty collections of variables. It follows that these functions are the characteristic functions of $2 \times$ MDS codes in Doob graphs, which, in their turns, can be decomposable or not. As a result, the characteristic function of any $2 \times$ MDS code is a sum of the characteristic functions of one, two, or more (up to $m+n$ ) indecomposable $2 \times$ MDS codes. With some natural assumptions, such a representation is unique:

Lemma 2 The characteristic function $\chi_{M}$ of any $2 \times M D S$ code $M \subset{ }_{\mathrm{v}} D(m, n)$ has a unique representation in the form

$$
\chi_{M}(\bar{x}, \bar{y}) \equiv \chi_{M_{1}}\left(\tilde{x}_{1}, \tilde{y}_{1}\right)+\ldots+\chi_{M_{k}}\left(\tilde{x}_{k}, \tilde{y}_{k}\right)+\sigma \bmod 2
$$

where

- $\bar{x}=\left(x_{1}, \ldots, x_{m}\right) \in \mathrm{Sh}^{m}$

- $\bar{y}=\left(y_{1}, \ldots, y_{n}\right) \in K^{n}$;

- $\tilde{x}_{i}=\left(x_{j_{i, 1}}, \ldots, x_{j_{i, m_{i}}}\right) \in \mathrm{Sh}^{m_{i}}, i=1, \ldots, k$;

$$
\begin{aligned}
& m=\sum_{1}^{k} m_{i} ; \\
& \{1, \ldots, m\}=\left\{j_{1,1}, \ldots, j_{1, m_{1}}, \ldots, j_{k, 1}, \ldots, j_{k, m_{k}}\right\} ;
\end{aligned}
$$

- $\tilde{y}_{j}=\left(y_{l_{i, 1}}, \ldots, y_{l_{i, n_{i}}}\right) \in K^{n_{i}}, i=1, \ldots, k$;

$$
n=\sum_{1}^{k} n_{i} \text {; }
$$$$
\{1, \ldots, n\}=\left\{l_{1,1}, \ldots, l_{1, n_{1}}, \ldots, l_{k, 1}, \ldots, l_{k, n_{k}}\right\}
$$

- for all $i \in\{1, \ldots, k\}$ it holds $m_{i}+n_{i} \geq 1$ and $M_{i}$ is an indecomposable $2 \times M D S$ code not containing the all-zero tuple;

- $\sigma \in\{0,1\}$.

In this notation, $M$ is indecomposable if and only if $k=1$.

Proof We first note that the graph structure is not essential in this lemma, because the adjacency is not used. Next, the statement of the lemma holds for any vertex set $M$, not only for a $2 \times$ MDS code. It was proven in [9, Lemma A.4], but formally, only for the case when all the arguments take their values from the same set (in our context, this is the case if $n=0$ ). However, this restriction is not essential for the proof, and that proof can be referred for the general case (rewriting all arguments here is not reasonable; they are not difficult but accurate arguing takes some place). Another way is to satisfy this restriction by some artificial trick, see the proof of Lemma 12 below.

Obviously, an $2 \times \operatorname{MDS}$ code $M$ is decomposable if and only if $k>1$ in the representation (2). 


\section{Linear $2 \times$ MDS codes and semilinear MDS codes}

We will say that a $2 \times \mathrm{MDS}$ code is linear if $k=m+n$ in the representation (2), i.e., all $M_{i}$ are $2 \times \mathrm{MDS}$ codes in Sh or $K$, and, moreover, the ones that are in Sh are not connected (Fig. 4(a)). It is easy to see that all linear $2 \times$ MDS codes in a given $D(m, n)$ are equivalent.

We will say that an MDS code is semilinear if it is a subset of a linear $2 \times$ MDS code. In the rest of this section, after auxiliary statements, we evaluate the number of semilinear MDS codes. We omit the proof of the next lemma as it is obvious.

Lemma 3 Let a characteristic function $\chi_{M}$ of a decomposable $2 \times M D S$ code $M$ has a decomposition

$$
\chi_{M}(\bar{x}, \bar{y}) \equiv \chi_{M_{1}}\left(\tilde{x}_{1}, \tilde{y}_{1}\right)+\chi_{M_{2}}\left(\tilde{x}_{2}, \tilde{y}_{2}\right)+\sigma \bmod 2
$$

where each $M_{i}, i=1,2$, as well as its complement, has $N_{i}$ components. Then $M$, as well as its complement, has $2 N_{1} N_{2}$ components.

Lemma 4 Let $M$ be a $2 \times M D S$ code in $D(m, n)$, and let $N$ be the number of components in $M$.

a) If $M$ is a linear $2 \times M D S$ code then $N=2^{2 m+n-1}$.

b) If $M$ is bipartite, then the number of $M D S$ codes included in $M$ is $2^{N}$.

Proof a) The statement follows by induction from Lemma 3 .

b) As follows from the hypothesis, the subgraph $D_{M}$ of $D(m, n)$ induced by $M$ is bipartite. By the definition of an MDS code, a part of $D_{M}$ is an MDS code. To choose a part of $D_{M}$, one should independently choose one of two parts of every connected components of $D_{M}$. So, the number of ways is $2^{N}$.

Lemma 5 The number of linear $2 \times M D S$ codes in $D(m, n)$ is $2 \cdot 3^{m+n}$.

Proof Every linear $2 \times \mathrm{MDS}$ codes can be represented as (2) where for each $i \in\{1,2, \ldots, k=m+n\}$ the set $M_{i}$ is a $2 \times$ MDS code in Sh equivalent to the one in Fig. 4 (a) or a $2 \times$ MDS code in $K$ and, moreover, $00 \notin M_{i}$. In any case, $M_{i}$ can be chosen in 3 ways. Since $\sigma$ can be chosen in 2 ways, we have totally $2 \cdot 3^{m+n}$ ways to specify a linear $2 \times \operatorname{MDS}$ code.

Lemma 6 The number of semilinear MDS codes in $D(m, n)$ is

$$
2 \cdot 3^{m+n} \cdot 2^{2^{2 m+n-1}}(1+o(1)) \quad \text { as } m+n \rightarrow \infty .
$$

Proof There are $2 \cdot 3^{m+n}$ linear $2 \times$ MDS codes (Lemma 5), each including $2^{2^{2 m+n-1}}$ semilinear MDS codes (Lemma 41). It remains to understand that almost every semilinear MDS code is included in only one linear $2 \times$ MDS code. One of simple explanations of this fact is that two different linear $2 \times \mathrm{MDS}$ codes $M^{\prime}, M^{\prime \prime}$ can include at most one common MDS code. Indeed each of $M^{\prime}, M^{\prime \prime}$ is a coset of a subgroup of $\left(Z_{4}^{2}\right)^{m} \times\left(Z_{2}^{2}\right)^{n}$, so the cardinality of their intersection cannot be larger than $\left|M^{\prime}\right| / 2=\left|M^{\prime \prime}\right| / 2=4^{2 m+n-1}$, which is the cardinality of an MDS code. 


\section{Main results}

We are now ready to formulate the main result of the paper, which will be proven in the next two sections.

Theorem 1 Every $M D S$ code in $D(m, n)$ is semilinear or reducible.

Note that 'or' is not 'xor' here; a reducible MDS code (11) can also be semilinear. This happens when both graphs ${ }^{1}$ of $f$ and $f^{\prime}$ are semilinear and, moreover, the representations (2) of the corresponding linear $2 \times \mathrm{MDS}$ codes have the same summand $\chi_{M^{*}}\left(x_{0}\right)$ corresponding to the dependent variable $x_{0}\left(M^{*}\right.$ can be $\{(0,1),(1,0)\},\{(0,1),(1,1)\}$, or $\{(1,0),(1,1)\}$, but it is the same for both $f$ and $\left.f^{\prime}\right)$.

Corollary 1 The number of $M D S$ codes in $D(m, n)$ has the form

$$
2 \cdot 3^{m+n} \cdot 2^{2^{2 m+n-1}}(1+o(1)) \quad \text { as } \quad m+n \rightarrow \infty .
$$

Proof By Lemma 6, the number of semilinear MDS codes is $2 \cdot 3^{m+n} \cdot 2^{2^{2 m+n-1}}(1+$ $o(1))$. The number of reducible MDS codes is asymptotically inessential comparing with this value (the arguments for the general case do not differ from those for $D(0, n)$, considered in [14]).

Corollary 1 improves the result of [11, where simpler arguments are used to establish the asymptotic of the logarithm of the number of MDS codes in $D(m, n)$.

\section{A key proposition}

In this section, we will prove the following proposition, which will be used in the proof of the main theorem.

Proposition $1 A 2 \times M D S$ code in $D(m, n),(m, n) \neq(1,0)$, is decomposable if and only if it is not connected.

The following fact is easy to check directly.

Lemma 7 Let $M$ and $M^{\prime}$ be two $2 \times M D S$ codes in Sh. Then, either $M$ and $M^{\prime}$ coincide, or they are are disjoint (i.e., $\left.M \cup M^{\prime}={ }_{\mathrm{v}} \mathrm{Sh}\right)$, or every component of $M$ intersects with every component of $M^{\prime}$.

The following partial case of Proposition 1 will be used as an auxiliary statement.

Lemma $8 A 2 \times M D S$ code $M$ in $D(m, n), m+n=2$, is decomposable if and only if it is not connected. 
Proof By Lemma 3 every decomposable $2 \times$ MDS code is not connected, which is the 'only if' part of the statement. For the 'if' part, we have to show that any $2 \times$ MDS code $M$ in $D(m, n), m+n=2$, is decomposable or connected.

The case of $D(0,2)=K \times K$ is trivial, as there are only two nonequivalent $2 \times$ MDS codes, one is decomposable, the other is connected.

The case of $D(1,1)=\mathrm{Sh} \times K$ is also simple. Denote $M_{y}=\left\{x \in{ }_{\mathrm{vSh}}\right.$ $(x, y) \in M\}$ for each $y \in \mathrm{v} K$. Then $M_{y}$ is a $2 \times$ MDS code in Sh. If for all $y^{\prime}, y^{\prime \prime}$ from $v K$ the sets $M_{y^{\prime}}$ and $M_{y^{\prime \prime}}$ are either coinciding or disjoint, then, readily, $M$ is decomposable. Assume that for some $y^{\prime}, y^{\prime \prime} \in \mathrm{v} K$ the sets $M_{y^{\prime}}$ and $M_{y^{\prime \prime}}$ are neither coinciding nor disjoint. By Lemma 7 , the corresponding 16 vertices of $M$ belong to the same component. Moreover, for every $y$ from $v K$, the set $M_{y}$ is neither coinciding nor disjoint with at least one of $M_{y^{\prime}}, M_{y^{\prime \prime}}$. It follows that $M$ is connected.

It remains to consider the case of $D(2,0)=\mathrm{Sh} \times \mathrm{Sh}$. For $a \in \mathrm{Sh}$, denote

$$
\begin{aligned}
& R_{a}=\left\{x_{1} \in \mathrm{Sh} \mid\left(a, x_{1}\right) \in M\right\} \text { and } a R_{a}=\left\{\left(a, x_{1}\right) \in M\right\}, \\
& L_{a}=\left\{x_{1} \in \mathrm{Sh} \mid\left(x_{1}, a\right) \in M\right\} \text { and } L_{a} a=\left\{\left(x_{1}, a\right) \in M\right\} .
\end{aligned}
$$

Consider the case when $L_{00}$ is equivalent to the $2 \times$ MDS code in Fig. 4(a) (the other two cases are considered similarly); without loss of generality, assume $L_{00}=\{00,01,02,03,20,21,22,23\}$. Now consider the component $\{00,01,02$, $03\}$ of $L_{00}$.

(i) If $R_{00}=R_{01}=R_{02}=R_{03}$, then for every $a$ from Sh, the set $\{00,01,02$, $03\}$ is a component of $L_{a}$ or $\bar{L}_{a}$; hence, by Lemma 7, $L_{a}=L_{00}$ or $\bar{L}_{a}=L_{00}$. In this case, readily, $M$ is decomposable.

(ii) So, without loss of generality we can assume that $R_{00}$ and $R_{01}$ are different. Since they intersect in (00) (indeed, $\left.(00,00),(01,00) \in L_{00} \subset M\right)$, Lemma 7 means that $00 R_{00}$ and $01 R_{01}$ lie in a one component of $M$. Next, $R_{02}$ intersects with $R_{00}$; hence, $02 R_{02}$ lies in the same component as $00 R_{00}$. Similarly, $03 R_{03}$.

By similar arguments, each of $S_{0}=00 R_{00} \cup 01 R_{01} \cup 02 R_{02} \cup 03 R_{03}, S_{1}=$ $10 R_{10} \cup 11 R_{11} \cup 12 R_{12} \cup 13 R_{13}, S_{2}=20 R_{20} \cup 21 R_{21} \cup 22 R_{22} \cup 23 R_{23}, S_{3}=$ $30 R_{30} \cup 31 R_{31} \cup 32 R_{32} \cup 33 R_{33}$ is a subset of a component of $M$, and it remains to show that this component is common for all the four sets. For example, let us show that it is common for $S_{0}$ and $S_{1}$. Indeed, since $R_{00}$ and $R_{01}$ are different, $R_{11}$ intersects with at least one of them; this means that there is an edge connecting $00 R_{00} \cup 01 R_{01}$ and $11 R_{11}$. Similarly, there are edges connecting $S_{0}$ and $S_{3}$. The same argument is applicable to $S_{2}$ and $S_{3}$, except for the case $R_{20}=R_{21}=R_{22}=R_{23}$. Excluding this case by the argument similar to (i), we find that $M$ consists of one component.

Lemma 9 Let $M$ be a $2 \times M D S$ code in $D(m, n)$, let $L$ be a multicomponent of $M$, and let $L_{1}$ be the set of vertices of $D(m, n)$ at distance 1 from $L$. Then $L_{1}$ is a multicomponent of $\bar{M}$.

Proof Clearly, $L_{1}$ is a subset of $\bar{M}$. It remains to show that for each $b \in L_{1}$ every $c \in \bar{M}$ adjacent to $b$ also belongs to $L_{1}$. By the definition of $L_{1}$, there is 
$a \in L$ adjacent to $b$. Assume that $a$ and $b$ differ in the $i$ th coordinate, while $b$ and $c$ differ in the $j$ th coordinate.

(*) We will show that there is $d \in L$ adjacent to $c$ and differing from $c$ in the $i$ th coordinate. If $i=j$ is a $K$-coordinate, then this claim is obvious (we can take $d=a$ ). If $i=j$ is a Sh-coordinate, then it is also easy to see. Assume $i \neq j$ and consider the subgraph $D$ (isomorphic to $D(0,2), D(1,1)$, or $D(2,0)$ ) corresponding to these two coordinates and containing the vertices $a, b$, and c. Let $M^{\prime}$ be the intersection of $M$ with this subgraph. We know that $M^{\prime}$ is a $2 \times$ MDS code in $D$ and hence, by Lemma 8 , it is connected or decomposable. In the first case, $M^{\prime} \subseteq L$ and $(*)$ is trivial. In the last case, the vertex $d$ coinciding with $c$ in all coordinates except the $i$ th one and coinciding with $a$ in the $i$ th coordinate must belong to $L$ (indeed, from the decomposability we have

$$
\begin{aligned}
& \chi_{M^{\prime}}(a)+\chi_{M^{\prime}}(b)+\chi_{M^{\prime}}(c)+\chi_{M^{\prime}}(d) \\
& \quad \stackrel{\text { (3) }}{=}\left(\chi_{M_{1}^{\prime}}\left(a_{i}\right)+\chi_{M_{2}^{\prime}}\left(a_{j}\right)+\sigma\right)+\left(\chi_{M_{1}^{\prime}}\left(c_{i}\right)+\chi_{M_{2}^{\prime}}\left(a_{j}\right)+\sigma\right) \\
& \quad+\left(\chi_{M_{1}^{\prime}}\left(c_{i}\right)+\chi_{M_{2}^{\prime}}\left(c_{j}\right)+\sigma\right)+\left(\chi_{M_{1}^{\prime}}\left(a_{i}\right)+\chi_{M_{2}^{\prime}}\left(c_{j}\right)+\sigma\right)=0 \bmod 2,
\end{aligned}
$$

hence $d \in M^{\prime}$; since $d$ and $a$ are adjacent, we also have $\left.d \in L\right)$.

So, $(*)$ holds, and $L_{1}$ consists of components of $\bar{M}$.

Corollary 2 In $D(m, n)$, an $2 \times M D S$ code is uniquely determined by any of its multicomponents. In other words, two $2 \times M D S$ codes with a common multicomponent coincide.

Proof Let $L_{0}$ be a multicomponent of an $2 \times \operatorname{MDS}$ code $M$. Define recursively $L_{i+1}$ as the set of vertices of $D(m, n)$ at distance 1 from $L_{i}$. Since the diameter of the graph is $2 m+n$ and noting that $L_{i} \subseteq L_{i+2}$, we find

$$
\mathrm{v} D(m, n)=\bigcup_{i=0}^{2 m+n} L_{i}=L_{2 m+n-1} \cup L_{2 m+n} .
$$

By Lemma 9, $L_{1}, L_{3}, L_{5}, \ldots \subseteq \bar{M}$ and $L_{0}, L_{2}, L_{4}, \ldots \subseteq M$. We conclude that $M=L_{2 m+n-1}$ or $M=L_{2 m+n}$, depending on the parity of $2 m+n$.

Lemma 10 Let $M$ be $a 2 \times M D S$ code in $D(m, n)$ where $m>0$ and $m+n>1$. Let, for some $a_{2}, \ldots, a_{m} \in \mathrm{vSh}_{\mathrm{V}}$ and $b_{1}, \ldots, b_{n} \in \mathrm{v} K$,

$$
M^{\prime}=\left\{\left(x_{1}, a_{2}, \ldots, a_{m}, b_{1}, \ldots, b_{n}\right) \in M \mid x_{1} \in \mathrm{vSh}\right\} .
$$

Then either all 8 elements of $M^{\prime}$ belong to the same component of $M$ or $M$ is decomposable and

$$
\begin{aligned}
& \chi_{M}\left(x_{1}, x_{2}, \ldots, x_{m}, y_{1}, \ldots, y_{n}\right) \\
& =\chi_{M^{\prime}}\left(x_{1}, a_{2}, \ldots, a_{m}, b_{1}, \ldots, b_{n}\right)+\chi_{M_{0}}\left(x_{2}, \ldots, x_{m}, y_{1}, \ldots, y_{n}\right)+1 \bmod 2
\end{aligned}
$$

for some $2 \times M D S$ code $M_{0}$. 
Proof For $\alpha=\left(\alpha_{2}, \ldots, \alpha_{m}\right) \in \mathrm{vSh}^{m-1}$ and $\left(\beta_{1}, \ldots, \beta_{n}\right) \in{ }_{\mathrm{v}} K^{n}$, denote $M_{\alpha, \beta}=$ $\left\{x_{1} \in \mathrm{vSh} \mid\left(x_{1}, \alpha_{2}, \ldots, \alpha_{m}, \beta_{1}, \ldots, \beta_{n}\right) \in M\right\}$. Clearly, $M_{\alpha, \beta}$ is a $2 \times$ MDS code in Sh. If $M_{a, b}$ is connected, where $a=\left(a_{2}, \ldots, a_{m}\right), b=\left(b_{1}, \ldots, b_{n}\right)$, then, trivially, $M^{\prime}$ lies in a component of $M$ and the statement holds.

Assume that $M_{a, b}$ is not connected and, moreover, $M^{\prime}$ intersects with two components of $M$. Without loss of generality, suppose $\left(00, a_{2}, \ldots, a_{m}, b_{1}, \ldots, b_{n}\right) \in$ $M$, i.e., $00 \in M_{a, b}$. Define the $2 \times \operatorname{MDS}$ code $M_{0}=\{(\alpha, \beta) \mid(00, \alpha, \beta) \in M\}$; in particular, $(a, b) \in M_{0}$. Our goal is now to prove (3), or, equivalently, to prove that $M$ coincides with the $2 \times \operatorname{MDS}$ code $T \subset \mathrm{v} D(m, n)$ :

$$
\begin{aligned}
& \chi_{T}\left(x_{1}, x_{2}, \ldots, x_{m}, y_{1}, \ldots, y_{n}\right) \\
& \quad=\chi_{M_{a, b}}\left(x_{1}\right)+\chi_{M_{0}}\left(x_{2}, \ldots, x_{m}, y_{1}, \ldots, y_{n}\right)+1 \bmod 2 .
\end{aligned}
$$

Let $L_{0}$ be the component of $M_{0}$ that contains $(a, b)$. For every $\left(a^{\prime}, b^{\prime}\right) \in M_{0}$ adjacent to $(a, b)$ we have $M_{a^{\prime}, b^{\prime}}=M_{a, b}$ (otherwise, by Lemma 7. $M^{\prime}$ lies in one component, contradicting our assumption). Similarly, $M_{a^{\prime}, b^{\prime}}=M_{a, b}$ for every $\left(a^{\prime}, b^{\prime}\right)$ from $L_{0}$. Now we see that the set

$$
L=\left\{\left(x_{1}, x_{2}, \ldots, x_{m}, y_{1}, \ldots, y_{n}\right): x_{1} \in M_{a, b},\left(x_{2}, \ldots, x_{m}, y_{1}, \ldots, y_{n}\right) \in L_{0}\right\},
$$

is a multicomponent of $T$ (4) and, moreover, is a subset of $M$. By Corollary 2, we have $M=T$.

Lemma 11 ([9, Corollary 4.2, Remark 4.3]) Let $G=K_{q}^{n}$ be the Cartesian product of $n$ copies of the complete graph $K_{q}$ of even order $q$. Let $M$ be a set of vertices of $G$ such that every clique of order $q$ contains exactly $q / 2$ elements of $M$. Then the subgraph $G_{M}$ of $G$ induced by $M$ is disconnected if and only if the characteristic function $\chi_{M}$ of $M$ is decomposable into the sum $\chi_{M}(z)=\chi_{M^{\prime}}\left(z^{\prime}\right)+\chi_{M^{\prime \prime}}\left(z^{\prime \prime}\right) \bmod 2$, where $M^{\prime} \subset{ }_{v} K_{q}^{n^{\prime}}, M^{\prime \prime} \subset{ }_{v} K_{q}^{n^{\prime \prime}}$, and $z^{\prime}$, $z^{\prime \prime}$ are nonempty disjoint collections of variables from $z=\left(z_{1}, \ldots, z_{n}\right)$ of length $n^{\prime}$ and $n^{\prime \prime}$ respectively, $n^{\prime}+n^{\prime \prime}=n$.

Lemma 12 Let $G^{*}=G^{*}(m, n)=K_{16}^{m} \times K_{4}^{n}$ be the Cartesian product of $m$ copies of the complete graph $K_{16}$ of order 16 and $n$ copies of the complete graph $K_{4}$ of order 4 . Let $M^{*}$ be a set of vertices of $G^{*}$ such that every clique $K$ maximal by inclusion (it follows that $|K|=4$ or $|K|=16$ ) contains exactly $|K| / 2$ elements of $M^{*}$. Then the subgraph $G_{M^{*}}^{*}$ of $G^{*}$ induced by $M^{*}$ is disconnected if and only if the characteristic function $\chi_{M^{*}}$ of $M^{*}$ is decomposable into the sum $\chi_{M^{*}}(z)=\chi_{M^{\prime}}\left(z^{\prime}\right)+\chi_{M^{\prime \prime}}\left(z^{\prime \prime}\right) \bmod 2$, where $M^{\prime} \subset{ }_{\mathrm{v}} G^{*}\left(m^{\prime}, n^{\prime}\right)$, $M^{\prime \prime} \subset{ }_{\mathrm{v}} G^{*}\left(m^{\prime \prime}, n^{\prime \prime}\right)$, and $z^{\prime}, z^{\prime \prime}$ are nonempty disjoint collections of variables from $z=\left(x_{1}, \ldots, x_{m}, y_{1}, \ldots, y_{n}\right)$.

Proof We map each vertex $\left(x_{1}, \ldots, x_{m}, y_{1}, \ldots, y_{n}\right)$ of $G^{*}$ to $4^{n}$ elements

$$
\left(x_{1}, \ldots, x_{m},\left(y_{1}, z_{1}\right), \ldots,\left(y_{n}, z_{n}\right)\right), \quad z_{i} \in\{0,1,2,3\}, \quad i=1, \ldots, n,
$$

which will be treated as vertices of $K_{16}^{m+n}$. Then $M^{*}$ is mapped into a set $M$ of vertices of $K_{16}^{m+n}$. It is easy to see that $M$ satisfies the hypothesis of Lemma11. Moreover, the subgraph $G_{M}$ is connected if and only if the subgraph $G_{M^{*}}^{*}$ is connected; and the characteristic function $\chi_{M}$ is decomposable if and only if $\chi_{M^{*}}$ is decomposable. Then, the statement follows from Lemma 11. 
Proof (of Proposition 1) Assume that $M$ is a $2 \times \operatorname{MDS}$ code in $D(m, n)$, where $(m, n) \neq(1,0)$. If $M$ is connected, then it is indecomposable by Lemma 3 .

Assume that $M$ induces a disconnected subgraph of $D(m, n)$. Since $K_{16}^{m} \times$ $K_{4}^{n}$ is obtained from $D(m, n)$ by adding some edges, the subgraph of $K_{16}^{m} \times K_{4}^{n}$ induced by $M$ can be connected or not.

If $M$ induces a disconnected subgraph of $K_{16}^{m} \times K_{4}^{n}$, then the statement follows from Lemma 12

It remains to consider the case when the subgraph of $K_{16}^{m} \times K_{4}^{n}$ induced by $M$ is connected. This means that some Shrikhande subgraph of $D(m, n)$ intersects with two components of $M$ (then, adding the absent edges in this subgraph connects these two components), in particular, $m \geq 1$. But then $M$ is decomposable by Lemma 10. This proves the proposition. (However, to make the situation clear, we can further note that the last case is contradictory: the decomposability of $M$ implies that the induced subgraph of $K_{16}^{m} \times K_{4}^{n}$ is not connected.)

\section{Proof of the main theorem}

Proof (of Theorem 11) In the case $m=0$, the statement coincides with the main theorem of [12. The case $(m, n)=(1,0)$ is trivial. Consider an MDS code $C$ in $D(m, n), m>0, m+n>1$. For $\alpha=\left(\alpha_{2}, \ldots, \alpha_{m}\right) \in \mathrm{vSh}^{m-1}$ and $\beta=\left(\beta_{1}, \ldots, \beta_{n}\right) \in{ }_{\mathrm{v}} K^{n}$, denote $C_{\alpha, \beta}=\left\{x_{1} \in \mathrm{v} \operatorname{Sh} \mid\left(x_{1}, \alpha_{2}, \ldots, \alpha_{m}, \beta_{1}, \ldots, \beta_{n}\right) \in\right.$ $C\}$.

Assume that for all $\alpha$ and $\beta$ the MDS code $C_{\alpha, \beta}$ is equivalent to the code shown in Fig. 2(a), i.e., one of $C_{00}=\{00,02,20,22\}, C_{01}=\{01,03,21,23\}$, $C_{10}=\{10,12,30,32\}, C_{11}=\{11,13,31,33\}$. Then, it is easy to see that $C$ is reducible:

$$
C=\left\{\left(y, \bar{y}^{\prime}, \bar{x}^{\prime}\right) \in D(m, n) \mid f(y)=f^{\prime}\left(y^{\prime}, x^{\prime}\right)\right\}
$$

for some $f^{\prime}: \mathrm{v} D(m-1, n) \rightarrow\{00,01,10,11\}$ and for $f:{ }_{v} D(1,0) \rightarrow\{00,01$, $10,11\}$ satisfying $f(y)=i j$ for every $y \in C_{i j}$.

Otherwise, we may assume without loss of generality that $C_{a, b}=\{00$, 02, 21, 23\} (Fig. 2(b)) for some $a$ and $b$. To utilize Proposition 11 we consider the $2 \times \operatorname{MDS}$ code $M=C \cup(C+(01,00, \ldots, 00))$. We will see that it is decomposable. Define the $2 \times \operatorname{MDS}$ code $M_{0}=\left\{\left(x_{2}, \ldots, x_{n}, y_{1}, \ldots, y_{n}\right)\right.$ $\left(00, x_{2}, \ldots, x_{n}, y_{1}, \ldots, y_{n}\right) \in C$ or $\left.\left(01, x_{2}, \ldots, x_{n}, y_{1}, \ldots, y_{n}\right) \in C\right\}$. In particular, $(a, b) \in M_{0}$. Similarly to $C_{\alpha, \beta}$, we denote

$$
M_{\alpha, \beta}=\left\{x_{1} \in \mathrm{Sh} \mid\left(x_{1}, \alpha_{2}, \ldots, \alpha_{m}, \beta_{1}, \ldots, \beta_{n}\right) \in M\right\} .
$$

In particular, $M_{a, b}=\{00,01,02,03,20,21,22,23\}$ (Fig. 4(a)).

For every $\left(a^{\prime}, b^{\prime}\right) \in M_{0}$ at distance 1 from $(a, b)$, the MDS code $C_{a^{\prime}, b^{\prime}}$ contains 01 and, moreover, is disjoint with $C_{a, b}=\{00,02,21,23\}$. There is only one such code, $C_{a^{\prime}, b^{\prime}}=\{01,03,20,22\}$. It follows that

$$
M_{a^{\prime}, b^{\prime}}=\{00,01,02,03,20,21,22,23\}=M_{a, b}
$$


Similarly, $M_{\alpha, \beta}=M_{a, b}$ for all $(\alpha, \beta)$ from the same connected component $L_{0}$ of $M_{0}$ as $(a, b)$. Now, we can apply the same argument as in the proof of Lemma 10, define

$$
T: \quad \chi_{T}=\chi_{M_{a, b}}\left(x_{1}\right)+\chi_{M_{0}\left(x_{2}, \ldots, x_{m}, y_{1}, \ldots, y_{n}\right)}+1 \bmod 2
$$

and

$$
L=\left\{\left(x_{1}, x_{2}, \ldots, x_{m}, y_{1}, \ldots, y_{n}\right): x_{1} \in M_{a, b},\left(x_{2}, \ldots, x_{m}, y_{1}, \ldots, y_{n}\right) \in L_{0}\right\} ;
$$

note that $L$ is a multicomponent of $T$ and a subset of $M$, and hence $M=T$ by Corollary 2 .

So, we have that $C$ is included in a decomposable $2 \times \operatorname{MDS}$ code $M$. Consider the decomposition (2) of $M$ into indecomposable $2 \times \operatorname{MDS}$ codes $M_{i}$, $i=1, \ldots, k$ :

$$
\chi_{M}(\bar{x}, \bar{y}) \equiv \chi_{M_{1}}\left(\tilde{x}_{1}, \tilde{y}_{1}\right)+\ldots+\chi_{M_{k}}\left(\tilde{x}_{k}, \tilde{y}_{k}\right)+\sigma \bmod 2, \quad k \geq 2 .
$$

By Proposition 1. all $M_{i}$ are connected, except some $2 \times$ MDS codes in Sh, which are equivalent to the code in Fig 4(a). Assume without loss of generality that the first $m^{\prime}$ codes $M_{1}, \ldots, M_{m^{\prime}}$ are not connected and that they correspond to the first $m^{\prime}$ variables $x_{1}, \ldots, x_{m^{\prime}}$. Since $M$ includes at least one MDS code $C$, all $M_{i}, i=1, \ldots, k$ are bipartite, as well as their complements. It follows that for each $i$ there is a latin coloring $f_{i}:{ } \mathrm{v} D\left(m_{i}, n_{i}\right) \rightarrow \mathrm{v} K$ such that $M_{i}=\left\{z \in \mathrm{v} D\left(m_{i}, n_{i}\right) \mid f_{i}(z) \in\{10,11\}\right\}$. Define the linear $2 \times$ MDS code $I$ by

$$
\begin{aligned}
\chi_{I}\left(x_{1}, \ldots, x_{m^{\prime}}, z_{1}, \ldots, z_{k-m^{\prime}}\right)= & \chi_{M_{1}}\left(x_{1}\right)+\ldots+\chi_{M_{m^{\prime}}}\left(x_{m^{\prime}}\right) \\
& +\chi_{\{10,11\}}\left(z_{1}\right)+\ldots+\chi_{\{10,11\}}\left(z_{k-m^{\prime}}\right)+\sigma .
\end{aligned}
$$

It is straightforward that

$M=\left\{(\bar{x}, \bar{y}) \in{ }_{\mathrm{v}} D(m, n) \mid\left(x_{1}, \ldots, x_{m^{\prime}}, f_{m^{\prime}+1}\left(\tilde{x}_{m^{\prime}+1}, \tilde{y}_{m^{\prime}+1}\right), \ldots, f_{k}\left(\tilde{x}_{k}, \tilde{y}_{k}\right)\right) \in I\right\}$.

Then, for every MDS code $S \subset I$, the MDS code

$$
\left\{(\bar{x}, \bar{y}) \in \mathrm{v} D(m, n) \mid\left(x_{1}, \ldots, x_{m^{\prime}}, f_{m^{\prime}+1}\left(\tilde{x}_{m^{\prime}+1}, \tilde{y}_{m^{\prime}+1}\right), \ldots, f_{k}\left(\tilde{x}_{k}, \tilde{y}_{k}\right)\right) \in S\right\}
$$

is a subset of $M$. Since $I$ and $M$ consist of the same number of components, they include the same number of MDS codes. In particular, $C$ is also representable in the form (5) for some $S$. If $m^{\prime}=m$ and $k=m+n$, then $C$ is semilinear. Otherwise, $k<m+n$ or $k=m+n$ and $m^{\prime}<m$, and $C$ is reducible.

Remark 1 Theorem 1 gives a recursive constructive description of MDS codes in $D(m, n)$. The class of semilinear MDS codes is constructive directly; the reducibility reduces the situation to the graphs of smaller diameter. In the current paper, we prove the case $m>0$, but it is important to understand that the case $m=0$ is necessary for the description because some factors in the decomposition can have only $K$-coordinates. It should also be noted that the approach used in this paper cannot be directly adopted to the case $m=0$. Existence of at least one Shrikhande coordinate is essential for our current 
proof. We use it to embed the MDS code into an $2 \times$ MDS code having at least four connected components. In that worst case, the original code is reduced to a code with smaller number of Shrikhande coordinates, the total number of coordinates remaining the same. If $m=0$, we still can apply the same technique to a $K$-coordinate, but in the worst case, the $2 \times$ MDS code would have only two connected components; the induced decomposition of the embedded MDS code would be trivial and would not imply the reducibility. The difficulties hidden in this case are solved in the papers [14, 9, 10, 12, containing the complete proof of Theorem 1 for $m=0$.

\section{MDS codes and $2 \times$ MDS codes as sets with extremal properties; $1 \frac{1}{2} \times$ MDS codes}

We already know that the MDS codes in a Doob graph are exactly the maximum (by cardinality) independent sets (actually, we take this property as the definition of the MDS codes). In this section, we show that the $2 \times$ MDS codes also meet some extremal property. Namely, they are exactly the sets with maximum edge boundary (cut). Moreover, the MDS codes and the $2 \times$ MDS codes define equitable 2-partitions of the Doob graph with minimum eigenvalue.

\subsection{The maximum cut}

The edge boundary (also known as cut) of a set $V$ of vertices of a graph $G=\left({ }_{\mathrm{v}} G,{ }_{\mathrm{E}} G\right)$ is the set of edges $\left\{\{v, w\} \in \mathrm{E}_{\mathrm{E}} G \mid v \in V, w \notin V\right\}$.

Proposition 2 (a) The maximum size of the edge boundary of a vertex set in $D(m, n)$ is $(2 m+n) 4^{2 m+n}$. (b) A vertex set $M$ has the maximum edge boundary if and only if it is a $2 \times M D S$ code.

Proof It is straightforward from the definition that a $2 \times$ MDS code has the edge boundary of size $(2 m+n) 4^{2 m+n}$. It remains to show the upper bound for the statement a) and the 'only if' part of b).

(a) $(2 m+n) 4^{2 m+n}$ is $2 / 3$ of the total amount of edges in $D(m, n)$. Since $D(m, n)$ is the Cartesian product of several copies of the Shrikhande graph Sh and several copies of the complete graph $K$ of order 4 , it is sufficient to show that the number of boundary edges in each of these two graphs cannot be larger than $2 / 3$ of the total amount of edges, i.e., $\frac{2}{3} \cdot 48=32$ in the case of Sh and $\frac{2}{3} \cdot 6=4$ for $K$. For $K$, this is trivial. For Sh, this follows from the fact that each triangle (complete subgraph of order 3 ) cannot have more than 2 boundary edges, while every edge belong to the same number of triangles.

(b) We first note that it is sufficient to prove the statement for the graphs Sh and $K$. Indeed, if we have a set $M$ whose edge boundary size is $2 / 3$ of the total amount of edges in $D(m, n)$, then the same proportion takes place in every Shrikhande or $K$ subgraph (otherwise, there is a contradiction with 


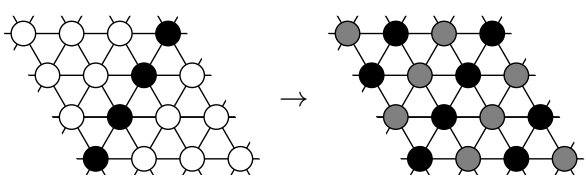

Fig. 5 There is a cycle of type 4:0

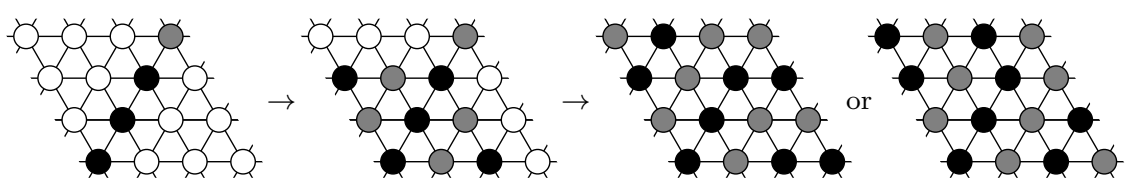

Fig. 6 There is a cycle of type 3:1

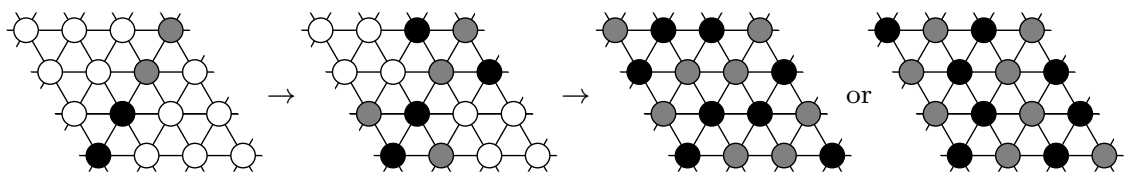

Fig. 7 All cycles are of type 2:2

p.(a) for such subgraphs). If we already have the statement for Sh and $K$, then $M$ is a $2 \times$ MDS code by the definition.

For $K$, it is trivial. Let us consider the Shrikhande graph and a vertex set $M$ with edge bound of size 32 . We color the vertices of $M$ by black and the other vertices by gray. From the previous paragraph we know that each triangle has at least one black and one gray vertex; we will always keep this fact in mind in the rest of the proof. The Shrikhande graph has 12 induced (i.e., without chords) 4-cycles. We consider three cases.

(4:0) There is an induced cycle colored into one color. Then, the colors of the other vertices are uniquely reconstructed (see Fig [5), leading to the situation shown in Fig. 4(a).

(3:1) There is a induced cycle with only one black vertex or only one grey vertex. The colors of six other vertices are uniquely reconstructed. Choosing the color of any of the six remaining vertices uniquely leads to one of two mutually symmetric colorings, see Fig 6. The result corresponds to Fig. 4(b).

(2:2) All induced cycles have two black and two gray vertices. Then, there is an induced cycle containing two neighbor black vertices, see Fig 7 , Four vertices out of this cycle are uniquely colored. Choosing the color of any of the eight other vertices and using only the hypothesis of the case $(2: 2)$, we uniquely color the seven remaining vertices, see Fig 7 Both $M$ and its complement are equivalent to the set shown in Fig. 4(c).

\subsection{Equitable partitions}

A partition $\left(P_{1}, \ldots, P_{r}\right)$ of the vertex set of a graph into $r$ nonempty cells is said to be an equitable partition, see e.g. [7, 9.3] (regular partition [2, 11.1B], 
partition design [3], perfect coloring [5]), if there is an $r \times r$ matrix $\left(s_{i j}\right)_{i, j=1}^{r}$ (the quotient matrix) such that for every $i$ and $j$ from 1 to $r$ every vertex from $P_{i}$ has exactly $s_{i, j}$ neighbors from $P_{j}$. It is known that each eigenvalue of the quotient matrix is an eigenvalue of the graph [2] (in a natural way, an eigenvector of the quotient matrix generates an eigenfunction of the graph). If the graph is regular, then its degree is always an eigenvalue of the quotient matrix.

The graph $D(m, n)$ has the $d+1$ eigenvalues $-d,-d+4, \ldots, 3 d$, where $d=2 m+n$ is the graph diameter. In $\mathrm{v} D(m, n)$, we consider an equitable 2-partition $(C, \bar{C})$ such that the quotient matrix has two eigenvalues $-d$ and $3 d$, the smallest and the largest eigenvalues of $D(m, n)$. This matrix has the form $\left[\begin{array}{cc}a & 3 d-a \\ a+d & 2 d-a\end{array}\right]$ for some $a$ from 0 to $2 d$. The cases $a=0, a=d$, and $a=2 d$ correspond to $C$ being an $M D S$ code, a $2 \times \operatorname{MDS}$ code, and the complement of an $M D S$ code.

Proposition 3 (a) A 2-partition $(C, \bar{C})$ of ${ }_{\mathrm{v}} D(m, n)$ is equitable with the quotient matrix $\left[\begin{array}{cc}0 & 6 m+3 n \\ 2 m+n & 4 m+2 n\end{array}\right]$ (or $\left[\begin{array}{cc}4 m+2 n & 2 m+n \\ 6 m+3 n & 0\end{array}\right]$ ) if and only if $C$ (respectively, $\bar{C})$ is an $M D S$ code. (b) A 2-partition $(M, \bar{M})$ of ${ }_{\mathrm{v}} D(m, n)$ is equitable with the quotient matrix $\left[\begin{array}{cc}2 m+n & 4 m+2 n \\ 4 m+2 n & 2 m+n\end{array}\right]$ if and only if $M$ is a $2 \times M D S$ code.

Proof We will use the formula $|S|=\frac{c}{b+c}|\mathrm{v} G|$ for the cardinality of the first cell $S$ of an equitable partition of a graph $G$ with the quotient matrix $\left[\begin{array}{ll}a & b \\ c & d\end{array}\right]$.

(a) For the first matrix, we have $a=0$; so, $C$ is an independent set. Counting its cardinality gives the cardinality of an MDS code. For the second matrix, similar argument works for $\bar{C}$.

(b) Count the size $|M| \cdot b$ of the edge boundary and apply Proposition 2 .

However, the cases $a=0, a=d$, and $a=2 d$ are not all possible cases. In $D(m, 0)$ (i.e., $d=2 m)$, the cases $a=0.5 d$ and $a=1.5 d$ are also feasible; that is there exists an equitable partition with the quotient matrix $\left[\begin{array}{cc}m & 5 m \\ 3 m & 3 m\end{array}\right]$. The first $(m=1)$ such partition is shown in Fig. 8 .

Proposition 4 Let $(C, \bar{C})$ be the partition shown in Fig. 8. Then

$$
\begin{aligned}
& \left(\left\{\left(x_{1}, \ldots, x_{m}\right) \in{ }_{\mathrm{v}} D(m, 0) \mid \sum_{i=1}^{m} x_{i} \in C\right\},\right. \\
& \left.\left\{\left(x_{1}, \ldots, x_{m}\right) \in{ }_{\mathrm{v}} D(m, 0) \mid \sum_{i=1}^{m} x_{i} \in \bar{C}\right\}\right)
\end{aligned}
$$

is an equitable partition with the quotient matrix $\left[\begin{array}{ll}1 m & 5 m \\ 3 m & 3 m\end{array}\right]$.

The proof is straightforward.

Remark 2 Equitable partitions with considered parameters are connected with unbalanced boolean functions of maximal correlation immunity, in the sense of the bound [4]. A function $f: \mathrm{v} H(N, 2) \rightarrow\{0,1\}$ is unbalanced if the number 

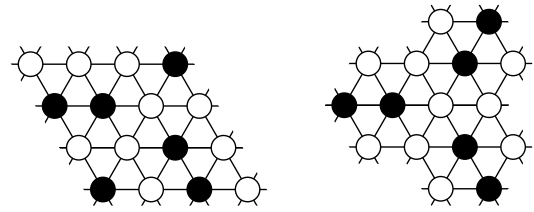

Fig. 8 An equitable 2-partition with quotient matrix $\left[\begin{array}{ll}1 & 5 \\ 3 & 3\end{array}\right]$

of its ones differs from $0,2^{N-1}$, and $2^{N}$, and is called a $k$ th order correlation immune if it has the same number of ones in every subgraph isomorphic to $H(N-k, 2)$. As was shown in [4, the unbalanced boolean functions with maximum correlation immunity order, $2 N / 3-1$, correspond to the equitable partitions of the hypercube graph $H(N, 2), N=3 d$, with quotient matrix $\left[\begin{array}{cc}a & 3 d-a \\ a+d & 2 d-a\end{array}\right], 3 d-a \neq a+d$. Only three classes such equitable partitions are currently known, they have $a=0, a=d / 2$, and $a=3 d / 4$ [6]. One can note that in the first two cases, the quotient matrices are the same as in Proposition 3(a) and Proposition 4 corresponding to the MDS-codes and " $1 \frac{1}{2} \times$ MDS codes". Indeed, there is there is a close connection between the considered classes of objects in $D(m, n)$ and in $H(6 m+3 n, 2)$ : with an inverse covering (locally isomorphic map from $\mathrm{v} H(6 m+3 n, 2)$ to $\mathrm{v} D(m, n))$ of $D(m, n)$ by $H(6 m+3 n, 2)$, any equitable partition of $D(m, n)$ is mapped to an equitable partition of $H(6 m+3 n, 2)$ with the same quotient matrix. Such covering can be easily constructed as a group homomorphism mapping a connecting set to a connecting set if we consider $H(6 m+3 n, 2)$ as a Cayley graph of $Z_{4}^{3 m} \times Z_{2}^{3 n}$ (the connecting set consists of the elements with only one nonzero coordinate, containing 1 or 3 ).

Remark 3 We briefly discuss a connection with another important concept related to the equitable partitions. A set $S$ of vertices of a graph is said to be completely regular (often, a completely regular code) if the partition of the graph vertices with respect to the distance from $S$ is equitable (the quotient matrix is tridiagonal in this case). The number of cells different from $S$ in this partition is called the covering radius of $S$. Trivially, each cell of an equitable 2 -partition is a completely regular set of covering radius 1 . It is interesting that not only MDS codes and $2 \times$ MDS codes, but in some cases the connected components of $2 \times \mathrm{MDS}$ codes are completely regular. As shown in [8], using the Cartesian product, from every completely regular code of covering radius 1 one can obtain a completely regular code of arbitrary covering radius. A component of a decomposable $2 \times \mathrm{MDS}$ code can be represented as the Cartesian product of $k 2 \times$ MDS codes, where $k$ is from (2). As follows from the results of $[8$, such a component is completely regular if and only if all $2 \times \operatorname{MDS}$ codes $M_{i}$, $i=1, \ldots, k$ in the decomposition (2) have the same quotient matrices. This happens if and only if $2 m_{1}+n_{1}=\ldots=2 m_{k}+n_{k}$. 


\section{Conclusion}

We have proven a characterization of the distance-2 MDS codes, or maximum independent sets, in the Doob graphs $D(m, n)$.

For related objects in $D(m, n)$, called the $2 \times$ MDS codes, we have shown the equivalence between the connectedness and the indecomposability. However, the problem of characterization of the $2 \times$ MDS codes (namely, those of them that cannot be split into two MDS codes) remains open. We have shown that the $2 \times$ MDS codes are exactly the sets with maximum cut (edge boundary).

We have noted that the MDS codes and the $2 \times \operatorname{MDS}$ codes in $D(m, n)$ are the equitable 2-partitions with certain quotient matrices. The eigenvalues of these matrices are the minimum and the maximum eigenvalues of $D(m, n)$. We have found that in the case $n=0$, there is a third class of equitable 2partitions that corresponds to these eigenvalues. Characterizing all partitions from this class is also an interesting direction for further research.

\section{References}

1. Bespalov, E.A., Krotov, D.S.: MDS codes in Doob graphs. Probl. Inf. Transm. 53(2), 136-154 (2017). DOI 10.1134/S003294601702003X. Translated from Probl. Peredachi Inf. 53(2), 40-59 (2017).

2. Brouwer, A.E., Cohen, A.M., Neumaier, A.: Distance-Regular Graphs. Springer-Verlag, Berlin (1989). DOI 10.1007/978-3-642-74341-2

3. Camion, P., Courteau, B., Delsarte, P.: On $r$-partition designs in Hamming spaces. Appl. Algebra Eng. Commun. Comput. 2(3), 147-162 (1992). DOI 10.1007/ BF01294330

4. Fon-Der-Flaass, D.G.: A bound on correlation immunity. Sib. Ehlektron. Mat. Izv. 4 133-135 (2007). URL http://mi.mathnet.ru/eng/semr149

5. Fon-Der-Flaass, D.G.: Perfect 2-colorings of a hypercube. Sib. Math. J. 48(4), 740-745 (2007). DOI 10.1007/s11202-007-0075-4. Translated from Sib. Mat. Zh. 48(4), 923-930 (2007)

6. Fon-Der-Flaass, D.G.: Perfect colorings of the 12-cube that attain the bound on correlation immunity. Sib. Ehlektron. Mat. Izv. 4, 292-295 (2007). URL http://mi.mathnet.ru/eng/semr158 In Russian. English translation: https://arxiv.org/abs/1403.8091

7. Godsil, C., Royle, G.: Algebraic Graph Theory. Springer (2001)

8. Koolen, J.H., Lee, W.S., Martin, W.J., Tanaka, H.: Arithmetic completely regular codes. Discrete Math. Theor. Comput. Sci. 17(3), 59-76 (2009)

9. Krotov, D.S.: On decomposability of 4-ary distance 2 MDS codes, double-codes, and $n$-quasigroups of order 4. Discrete Math. 308(15), 3322-3334 (2008). DOI 10.1016/j. disc.2007.06.038

10. Krotov, D.S.: On reducibility of $n$-ary quasigroups. Discrete Math. 308(22), 5289-5297 (2008). DOI 10.1016/j.disc.2007.08.099

11. Krotov, D.S.: On the number of maximum independent sets in Doob graphs. Sib. Ehlektron. Mat. Izv. 12, 508-812 (2015). DOI 10.17377/semi.2015.12.043

12. Krotov, D.S., Potapov, V.N.: $n$-Ary quasigroups of order 4. SIAM J. Discrete Math. 23(2), 561-570 (2009). DOI 10.1137/070697331

13. Potapov, V.N.: On extensions of partial $n$-quasigroups of order 4. Sib. Adv. Math. 22(2), 135-151 (2012). DOI 10.3103/S1055134412020058. Translated from Mat. Tr. 14(2), 147-172 (2011)

14. Potapov, V.N., Krotov, D.S.: Asymptotics for the number of $n$-quasigroups of order 4 . Sib. Math. J. 47(4), 720-731 (2006). DOI 10.1007/s11202-006-0083-9. Translated from Sib. Mat. Zh. 47(4), 873-887 (2006) 